\title{
A standard ventriculoperitoneal shunt placement complicated by tension pneumocephalus without an identified trigger
}

Marek Prokopienko, Michał R. Sobstyl

Department of Neurosurgery, Institute of Psychiatry and Neurology, Warsaw, Poland

Submitted: 21 February 2021

Accepted: 2 June 2021

Arch Med Sci Aging 2021; 4: e23-e26

DOI: https://doi.org/10.5114/amsa.2021.107805

Copyright $\odot 2021$ Termedia \& Banach

\author{
Corresponding author: \\ Dr. Marek Prokopienko \\ Department of \\ Neurosurgery \\ Institute of Psychiatry \\ and Neurology \\ Warsaw, Poland \\ E-mail: mpnchir@gmail.com
}

Tension pneumocephalus (TP) develops in most cases as a result of head trauma. This phenomenon has also been described in patients with intracranial neoplasms, and after cranial base and paranasal sinus surgical procedures. TP may be a complication of a nontraumatic spontaneous cerebrospinal fluid (CSF) rhinorrhea after successful ventriculoperitoneal (VP) shunt placement due to congenital cranial base fistula. TP complication has been reported after lumbar puncture, spinal anesthesia or CSF drainage via a lumbar spinal catheter. In the above-mentioned instances, the provoking factors for a TP caused by the connection of the intracranial space and external environment have been well established. We report a case of a TP after a VP shunt placement in a patient with normal pressure hydrocephalus (NPH). The patient we describe had no history of cerebrospinal nontraumatic rhinorrhea, or head trauma. Computed tomography (CT) of the anterior fossa and petrous bones revealed no defects. The chest and abdominal CT were unremarkable. Due to progressive enlargement of the pneumocephalus the decision to remove the VP shunt was made, with a good clinical postoperative outcome. As the remission of TP in the postoperative course occurred without further VP shunt dependency, the situation is unique as it has not been described so far.

This 71-year-old patient was admitted to the Neurological Clinic of the Institute of Psychiatry and Neurology in Warsaw with suspected ischemic stroke due to right-sided hemiparesis and slight dysarthria. Deterioration of the patient's state was possibly caused by dehydration which clinically presented as transient ischemic attack. After intravenous administration of fluids, the patient's condition improved significantly. During hospitalization, the classical symptoms of NPH were established. The family confirmed the presence of wide-based gait, urinary incontinence, and memory deterioration for the last months. CT brain scans confirmed widening of the cerebral ventricles (Figure $1 \mathrm{~A}$ ). The neuropsychological assessment confirmed typical deficits observed in patients with NPH. As the CSF tap test and lumbar infusion test could not be performed due to iatrogenic blood coagulation disorders, the NPH diagnosis was made on the basis of the clinical and radiological (CT) picture.

The patient was referred for VP shunt placement. After signing the informed consent, the right medium-pressure VP shunt was implanted in the Neurosurgical Department of the Institute of Psychiatry and Neurology. The surgery was performed in January 2018 without compli- 

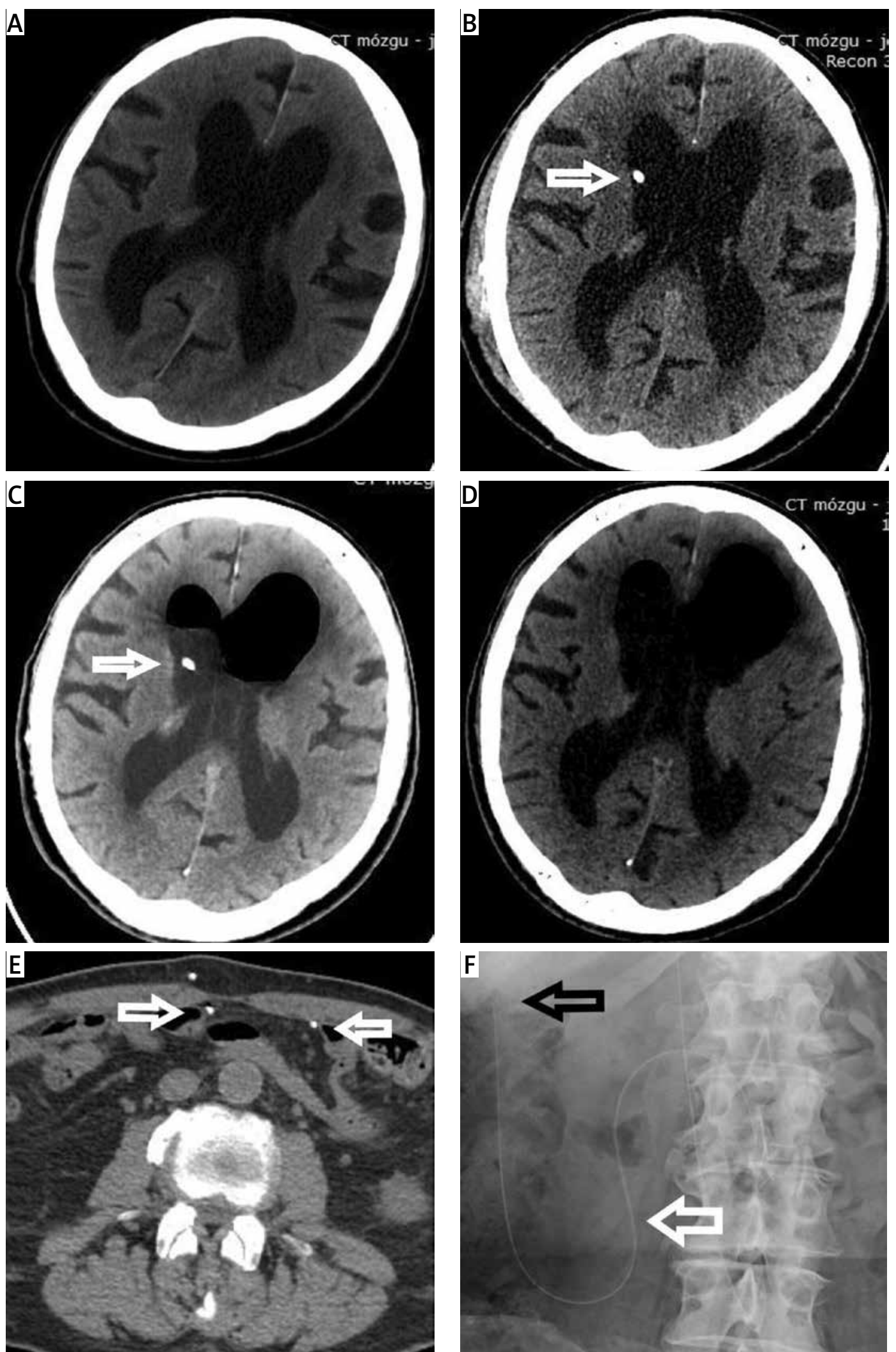

Figure 1. A - Computed tomography (CT) scan before ventriculoperitoneal shunt implantation showing enlargement of the ventricular system. B - Postoperative CT scan demonstrating the ventricular catheter (white arrow) placed in the right frontal horn. C, D - CT scans with tension pneumocephalus. Frontal horns are filled with air and are more enlarged when compared to preoperative $\mathrm{CT}$ indicating tension pneumocephalus. Left frontal horn is more widened than the right, which may be responsible for the patient's dysarthria. E - Abdominal CT showing proper peritoneal catheter location without evidence of air in the abdominal cavity. $\mathbf{F}-\mathrm{X}$-ray also excluded the presence of air in the area of the peritoneal catheter (white arrow) and its tip (black arrow) in the peritoneum 
cations (Figure $1 \mathrm{~B}$ ). The patient was discharged home with significant improvement on the third postoperative day. Three months after surgery the patient was fully independent.

In August 2019, the patient was admitted again to the Neurological Clinic due to right-sided paresis and signs of dysarthria. The CT scans showed widening of the ventricular system and TP in both frontal horns and the temporal horn of the left lateral ventricle (Figures 1 C, D). The presence of TP suggested dysfunction of the VP shunt. The patient and his family members denied any head injury. The inspection of the skin above the shunt system revealed no signs of damage. Abdominal CT examination and X-ray of the abdomen showed the preserved continuity of the drain, without signs of free gas in the peritoneum. Also the chest X-ray was unremarkable. Exploratory laparoscopy showed no intestinal perforation; the drain was placed in the peritoneum outside the bowels. CSF was collected and cultured without microbial growth. Its general examination showed no abnormalities.

As the CT scans of the brain revealed ventricular air volume progression, the shunt system was removed. After the procedure, the patient's general and neurological status improved. The patient became shunt-independent. The tension pneumocephalus and NPH resolved spontaneously. Follow-up CT scans revealed significant ventricular air volume reduction. The patient was discharged home in good clinical condition. Nearly 12 months after shunt removal the patient's general condition is stable without clinical signs of $\mathrm{NPH}$.

Since Chiari in 1884 reported that air can enter the ventricular system through the ethmoidal cells [1], the most common site of fistula between the intracranial space and the external environment has been established. The advances in medicine have created tools to alter intracranial pressure levels, which caused the activation of many, yet not occult cranial base fistulas. Cases of TP complicating ventriculoperitoneal shunt implantation have been continuously described for over 40 years [2, 3]. In non-traumatic patients with active hydrocephalus, the connection between the intracranial cavity and the atmosphere can be possible throughout sites of bony erosion caused by chronically elevated intracranial pressure. Pneumocephalus occurs when the intracranial pressure drops below the atmospheric pressure. This situation happens mostly after low-pressure shunt implantation. The negative intracranial pressure produced by the siphon effect of the shunt works like a vacuum, sucking air through the cranial base defect. In most of the cases the location of the fistula can be found in the anterior cranial fossa, as the bony layer in this location is very thin [2-7].
The lamina cribrosa of the ethmoidal bone is the most vulnerable site. The second most common fistula location is the tegmen tympani [8-12]. The incidence of cranial base defect at this site is nearly as high as in the area of the lamina cribrosa. The frontal sinus wall is a less common site of the leak $[13,14]$. In this situation endoscopic diagnosis and repair should be considered. Nontraumatic tension pneumocephalus occasionally can be caused by a scalp fistula from a remote ventriculoperitoneal shunt [15]. In this case percutaneous aspiration of the tension pneumocephalus can be stopped by fistula closure. Pneumocephalus can also be associated with lumbar puncture [16, 17], and migration of the peritoneal drain into the bowel $[18,19]$.

As proper functioning of the ventricular shunt is indispensable in most cases, the main aim of treatment is anterior fossa fistula closure, with preservation of the ventricular shunt $[3,5]$. Comprehensive treatment must be divided into stages. In the first stage, the shunt has to be removed. Then the cranial base fistula must be closed and finally shunt replaced at the different location [4]. In the case of lamina cribrosa defects, restoration of the cranial base surface should be as wide as possible, as it is sometimes difficult to locate precisely the site of the fistula. Repair of middle ear defects also prevents pneumocephalus recurrence, as the mechanism of air inflow is the same as in the case of anterior skull base fistulas. Careful inspection of the entire petrous surface is necessary.

In our case, a cranial base fistula was not found. The inspection of the skin above the implanted shunt revealed no signs of damage. The peritoneum revealed no air around the peritoneal shunt with subsequent laparoscopy showing its correct position. As the patient was no longer shunt dependent, after VP shunt removal, the volume of intraventricular air significantly decreased and further neurosurgical treatment was not necessary. As we based our practice on the experience of other authors [4, 6, 14], implantation of a high-pressure valve with an antisiphon device was planned even in the case of precise location and closure of the fistula site.

Fortunately, the patient has remained in a good clinical condition up to the present date (12 months after VP shunt removal). To our knowledge this is the first reported case of a tension pneumocephalus without an identified trigger. Moreover, the further postoperative course after VP shunt removal was unusual, because of spontaneous remission of TP without VP shunt dependency progression. That is why, in such cases, caution should always be exercised when considering VP shunt implantation prior to the onset of $\mathrm{NPH}$ symptoms. 


\section{Conflict of interest}

The authors declare no conflict of interest.

\section{References}

1. Chiari H. Ueber einen Fall von Luftansammlung in den Ventrikeln des meschlichen Gehirns. Z Heilk 1884; 5: 383-90.

2. Czepko R, Morga R, Uhl H. Pneumocephalus after shunt in a patient with hydrocephalus. A case report and review of the literature. Neurol Neurochir Pol 2005; 39: 524-30.

3. Ikeda K, Nakano M, Tani E. Tension pneumocephalus complicating ventriculoperitoneal shunt for cerebrospinal fluid rhinorrhoca: case report. J Neurol Neurosurg Psychiatry 1978; 41: 319-22.

4. Villarejo F, Carceller F, Alvarez C, et al. Pneumocephalus after shunting for hydrocephalus. Child's Nerv Syst 1998; 14: 333-7.

5. Schechter MM, Rovit RL, Schachter JM. Rhinorrhea and hydrocephalus. Observations in patients with increased intracranial pressure. Acta Radiologica Diagnosis 1969; 9: 101-16.

6. Cantisani PL, Cancelliere M, Armenise B, Lupo F. Hypertensive pneumocephalus and nasal fistula in ventriculo-peritoneal shunt: case report and review of literature. J Neurosurg Sci 1999; 43: 153-7.

7. Najera Aguilar E, Castle Ramirez M, Bollar Zabala A, Urculo Bareño E. Delayed spontaneous pneumocephalus in ventriculoperitoneal shunting: two case reports and literature review. Neurocirugia 2014; 25: 86-9.

8. Goffin J, Plets C. Tension pneumocephalus in association with ventriculoperitoneal shunt. Acta Neurochir 1985; 76: 121-4.

9. Pitts LH, Wilson CB, Dedo HH, Weyand R. Pneumocephalus following ventriculoperitoneal shunt. Case report. J Neurosurg 1975; 43: 631-3.

10. Salem-Memou S, Vallee B, Jacquesson T, Jouanneau E, Berhouma M. Pathogenesis of delayed tension intraventricular pneumocephalus in shunted patient: possible role of nocturnal positive pressure ventilation. World Neurosurg 2016; 85: 365.e17-20.

11. Verhaeghe A, De Muynck S, Casselman JW, Vantomme N. Delayed intraventricular pneumocephalus following shunting for normal-pressure hydrocephalus. World Neurosurg 2018; 116: 174-7.

12. Martinez-Perez R, Gómez E, Rayo N. Spontaneous tension pneumocephalus: a rare complication of shunting. World Neurosurg 2017; 100: 710.e11-3.

13. Sankhla S, Khan GM, Khan MA. Delayed tension pneumocephalus: a rare complication of shunt surgery. Neurol India 2004; 52: 401-2.

14. Honeybul S, Bala A. Delayed pneumocephalus following shunting for hydrocephalus. J Clin Neurosci 2006; 13: 939-42.

15. Monas J, Peak DA. Spontaneous tension pneumocephalus resulting from a scalp fistula in a patient with a remotely placed ventriculoperitoneal shunt. Ann Emerg Med 2010; 56: 378-81.

16. Kozikowski GP, Cohen SP. Lumbar puncture associated with pneumocephalus: report of a case. Anesth Analg 2004; 98: 524-6.

17. Gonzalez-Carrasco FJ, Aguilar JL, Llubia C, et al. Pneumocephalus after accidental dural puncture during epidural anesthesia. Reg Anesth 1993; 18: 193-5.
18. Harischandra LS, Sharma A, Chatterjee S. Shunt migration in ventriculoperitoneal shunting: a comprehensive review of literature. Neurol India 2019; 67: 85-99.

19. Jea A, Baskaya MK, Farhat H, Benglis D, Zauner A. Pneumocephalus in a patient with a ventriculoperitoneal shunt after percutaneous gastrojejunostomy catheter placement: case report. Surg Neurol 2006; 65: 87-9. 